Pp. 1-7

DOI: $10.1089 / \mathrm{gtmb} .2017 .0048$

\title{
Synergistic Association of Genetic Variants with Environmental Risk Factors in Susceptibility to Essential Hypertension
}

\author{
Ana Célia Sousa, Maria I. Mendonça, Andreia Pereira, Sara Gouveia,, Ana I. Freitas,,2 \\ Graça Guerra,,2 Mariana Rodrigues, Eva Henriques,' Sónia Freitas, Sofia Borges, \\ Décio Pereira, António Brehm, and Roberto Palma dos Reis ${ }^{3}$
}

Aims: Essential hypertension (EH) is a disease in which both environment and genes have an important role. This study was designed to identify the interaction model between genetic variants and environmental risk factors that most highly potentiates EH development.

Methods: We performed a case-control study with 1641 participants (mean age 50.6 \pm 8.1 years), specifically 848 patients with EH and 793 controls, adjusted for gender and age. Traditional risk factors, biochemical and genetic parameters, including the genotypic discrimination of 14 genetic variants previously associated with $\mathrm{EH}$, were investigated. Multifactorial dimensionality reduction (MDR) software was used to analyze geneenvironment interactions. Validation was performed using logistic regression analysis with environmental risk factors, significant genetic variants, and the best MDR model.

Results: The best model indicates that the interactions among the ADD1 rs4961 640T allele, diabetes, and obesity (body mass index $\geq 30$ ) increase approximately four-fold the risk of $\mathrm{EH}$ (odds ratio $=3.725 ; 95 \%$ confidence interval: $2.945-4.711 ; p<0.0001)$.

Conclusion: This work showed that the interaction between the ADD1 rs4961 variant, obesity, and the presence of diabetes increased the susceptibility to $\mathrm{EH}$ four-fold. In these circumstances, lifestyle adjustment and diabetes control should be intensified in patients who carry the ADDl variant.

Keywords: genes, $A D D 1$, environmental risk factors, obesity, MDR software, diabetes

\section{Introduction}

$\mathbf{E}$ SSENTIAL HYPERTENSION (EH) is a complex and multifactorial disease resulting from multiple environmental and genetic factors (Carretero and Oparil, 2000; Kuneš and Zicha, 2009; Joseph et al., 2013).

Both clinical and experimental studies have already identified plausible mechanisms underlying the development of $\mathrm{EH}$ : increased activity of the sympathetic nervous system, overactivity of the renin-angiotensin aldosterone system, dysfunction of the vascular endothelium, thrombogenesis, and impairment of the pressure-natriuresis mechanism leading to salt retention and elevated peripheral resistance, among others (Oparil et al., 2003). All these systems are under strict genetic control, and this is the cause that some individuals are more susceptible to EH than others.
The majority of currently available knowledge has greatly benefited by mapping the genes responsible for Mendelian forms of hyper- and hypotension (Lifton et al., 2001) and studying rodent models with various blood pressure (BP) affecting phenotypes (Okamoto and Aoki, 1963; Cowley, 2006). These association studies have successfully identified multiple interacting molecular pathways that are involved in the determination of a subject's BP. Consequently, genes coding for the components of these molecular pathways have been targeted for the identification of genetic variations affecting interindividual differences in BP levels (Mein et al., 2004; Ji et al., 2008). However, results of a large number of association studies conducted with BP traits have been inconsistent.

Genome-wide association studies (GWAS) have emerged as a novel alternative to explore simultaneously a large number of genomic loci for associations with a phenotypic trait. This

\footnotetext{
${ }^{1}$ Unidade de Investigação, Hospital Doutor Nélio Mendonça, Funchal, Portugal.

${ }^{2}$ Laboratório de Genética Humana, Universidade da Madeira, Funchal, Portugal.

${ }^{3}$ Faculty of Medical Sciences, New University of Lisbon, Lisboa, Portugal.
} 
method has shown a great promise in determining common polymorphisms responsible for several complex phenotypes like diabetes, stroke, and coronary artery disease (Altshuler et al., 2008). However, GWAS have not produced clear results in respect of hypertension-associated gene polymorphisms (Wellcome Trust Case Control Consortium, 2007; Levy et al., 2007; Ehret et al., 2008). Although it has identified numerous loci associated with BP traits (Levy et al., 2009), they only explain a small proportion of interindividual BP variability. The 29 loci identified by GWAS and reported by the International Consortium of Blood Pressure accounts for about $1 \%$ of $\mathrm{BP}$ variation in the general population (Ehret et al., 2011).

Knowing the universal nature of epistasis in determining susceptibility to complex disease, one possible way to approach these complex conditions is to examine the joint impact of multiple genes involved in particular cellular or physiological pathways along with several environmental risk factors underlining it. Moreover, many clinical and experimental studies have bypassed important environmental factors that can affect BP development and progression (Zuoguang et al., 2013).

In these circumstances, in complex diseases, like $\mathrm{EH}$, more than to evaluate the polymorphisms one by one, it is important to evaluate several polymorphisms together with environmental factors.

\section{Objective}

The goal of the current study is to evaluate the best interaction model between 14 single nucleotide polymorphisms (SNPs) associated to $\mathrm{EH}$ and several environmental risk factors, in predicting the risk of $\mathrm{EH}$ in the population of Madeira Island (Portugal).

\section{Methods}

\section{Study population}

All subjects participating in the present study were selected from the Internal, General, and Familiar Medicine from our Hospital.

This study received approval from the ethics committee of the Funchal Hospital Center and was performed in conformity with the guidelines outlined in the Declaration of Helsinki statement. Written informed consent was obtained from each participant, including explicit permission for the DNA analyses and the collection of relevant clinical data.

\section{Study design}

A case-control study was performed with a cohort of 1641 individuals (50.6 \pm 8.1 years; $49.9 \%$ male), namely 848 patients diagnosed with $\mathrm{EH}$ (mean age $50.8 \pm 8.1 ; 51.8 \%$ male) and 793 normotensive controls (mean age of $50.3 \pm 8.2 ; 47.9 \%$ male). Cases and controls were matched for sex and age.

$\mathrm{EH}$ was considered when patients, at the entry into this study, were already diagnosed and/or had been on antihypertensive medication for more than 3 months or newly diagnosed hypertensives with systolic blood pressure (SBP)/ diastolic blood pressure (DBP) $\geq 140 / 90 \mathrm{mmHg}$ measured on at least three occasions (European Society of HypertensionEuropean Society of Cardiology Guidelines Committee, 2003). The normotensive controls had never been treated with antihypertensive medication and presented a SBP/DBP $<140 / 90 \mathrm{mmHg}$.

BP was measured after 10 min of resting in the right arm, using a standard Welch Allyn sphygmomanometer (phases I through V). The average of three readings taken 2 min apart was recorded (European Society of Hypertension-European Society of Cardiology Guidelines Committee, 2003).

\section{Data collection}

Data recorded from each subject comprised demographic, clinical profile, and traditional risk factors (gender, age, body mass index [BMI], sedentary lifestyle, alcohol and smoking habits, and diabetes).

BMI was defined as body mass divided by the square of the body height, universally expressed in units of $\mathrm{kg} / \mathrm{m}^{2}$, with obesity defined as a BMI $\geq 30$ (National Institutes of Health, National Heart, Lung, and Blood Institute North American Association for the Study of Obesity, 2000). Sedentary lifestyle was considered when individuals practiced $<150 \mathrm{~min} /$ week of moderate activity or $75 \mathrm{~min}$ of vigorous activity) (The Department of Health, Government of Australia, 2014). Alcohol consumption was considered as a risk factor when individuals consumed $70 \mathrm{~g}$ of alcohol per week for more than 1 year. Moreover, individuals who smoked $\geq 70$ cigarettes per week for more than 1 year were defined as "smokers" (Ji et al., 2013).

Subjects were classified as being diabetic when taking oral antidiabetic medication or insulin or if their fasting plasma glucose was higher than $7.0 \mathrm{mmol} / \mathrm{L}$ or $126 \mathrm{mg} / \mathrm{dL}$ (Expert Committee on the Diagnosis and Classification of Diabetes Mellitus, 2003).

\section{Biochemical analysis}

Blood samples were extracted after 14-16 h fasting. Biochemical analyses were performed in the Central Laboratory of the Hospital, according to standard techniques. To determine total cholesterol, high-density lipoprotein (HDL), lowdensity lipoprotein, triglycerides, and glucose, blood samples were placed in dry tubes, centrifuged half an hour later at $3500 \mathrm{~g}$ and subsequently quantified by an enzymatic technique using a "AU 5400" (Beckman Coulter) auto analyzer. Biochemical markers such as lipoprotein-a, apolipoprotein B, and high-sensitivity $\mathrm{C}$-reactive protein (hs-CRP) were quantified by immunoturbidimetry using an AU 5400 (Beckman Coulter) automatic system. To measure fibrinogen, samples were placed in a tube containing sodium citrate, and measurements were taken with an ACL TOP 700 automatic analyzer.

\section{SNP selection}

SNPs were selected either from GWAS or candidate gene association studies for loci biologically plausible to $\mathrm{EH}$ or BP, specifically: ACE rs4340, ACE rs4343, AT1R rs5186, AGT rs699, AGT rs4762, ADD1 rs4961, CYP11B2 rs1799998, CYP17A1 rs11191548, $A D R \beta 1$ rs1801253, $A D R \beta 2$ rs1042713, $G N \beta 3$ rs5443, AT2B1 rs2681472, SLC4A2 rs2303934, and SCNN1G rs5718.

Entering criteria included genes with a minor allele frequency $(\mathrm{MAF})>5 \%$. Genes in Hardy-Weinberg disequilibrium ( $p<0.0036$, after Bonferroni correction) were automatically excluded. 


\section{Genetic analyses}

Genetic analyses were done at the Human Genetics Laboratory of the University of Madeira. Genomic DNA was extracted from $80 \mu \mathrm{L}$ of peripheral blood using a standard phenol-chloroform method. A TaqMan allelic discrimination assay for genotyping was performed using labeled probes and primers pre-established by the supplier (TaqMan SNP Genotyping Assays; Applied Biosystems).

Quality check of TaqMan genotyping technique was maintained by the inclusion of one nontemplate control in each plate of 96 wells. Also, all SNP TaqMan assays had blind duplicates accounting for $20 \%$ of all samples. Some SNP genotypes were randomly confirmed by conventional direct DNA sequencing, as $10-15 \%$ of all samples were reamplified for sequencing.

All reactions were done on an Applied Biosystems 7300 RealTime PCR System and genotypes were determined using the 7300 System SDS Software (Applied Biosystems, Foster City, CA) without any prior knowledge of individual's clinical data.

\section{Statistical analyses}

Genotypic frequencies were determined from observed counts and compared by Chi-square analysis. Representative samples were judged by comparing the genetic polymorphism frequency and Hardy-Weinberg equilibrium was tested at each locus on a contingency table of observed versus predicted genotype using the Chi-squared test.

Continuous and categorical variables were compared between the two groups by the unpaired Student's $t$-test, Mann-Whitney, and the Chi-squared test when appropriate. A two-tailed $p$ value of $<5 \%$ was considered statistically significant, whereas a value of $\mathrm{Pb}$ (PBonferroni divided by total number of comparisons) was considered significant after the Bonferroni correction.

Five statistical genetic models were tested, namely dominant, recessive, additive, multiplicative, and codominant. The relative risk factor was evaluated using odds ratio (OR) and $95 \%$ confidence interval (CI) for each model.

Interactions between variants significantly associated with $\mathrm{EH}$ and five environmental risk factors for $\mathrm{EH}$ (sedentary lifestyle, BMI, diabetes, smoking, and alcohol habits) were analyzed by using multifactorial dimensionality reduction (MDR) 3.0.2 software (Guy et al., 2010; Yang et al., 2015). The best combination pattern was searched on the principle that both cross-validation consistency and test balance accuracy were maximized to evaluate the interaction of the genetic polymorphism with each other and with the five environmental risk factors, in relation to $\mathrm{EH}$.

To test the validity of MDR method, we further conducted a classical logistic regression analysis that included the five environmental risk factors and the best MDR model.

Statistical analyses were performed using the Statistical Package for the Social Sciences Software version 19.0 (IBM, Armonk, NY).

\section{Results}

\section{Characteristics of the population}

Baseline characteristics of our population are listed in Table 1. There were no significant differences between cases and controls in terms of age and sex. When compared with controls, patients with EH had higher alcohol consumption $(p=0.008)$, BMI $(p<0.0001)$, obesity $(p<0.0001)$, diabetes $(p<0.0001)$, $\operatorname{SBP}(p<0.0001), \operatorname{DBP}(p<0.0001)$, and heart rate $(p<0.0001)$ (Table 1). Controls had more smoking habits in relation to hypertensive subjects, with statistical significance $(p<0.0001)$.

Biochemical analysis of the population is shown in Table 2. Comparatively to controls, hypertensive patients had significantly higher levels of hemoglobin $(p<0.0001)$, leucocytes $(<0.0001)$, fibrinogen $(<0.0001)$, glucose $(<0.0001)$, triglycerides $(<0.0001)$, apolipoprotein B $(p<0.0001)$, and hs-CRP $(<0.0001)$. On the other hand, HDL cholesterol showed higher values in controls, with a $p<0.0001$ (Table 2).

\section{Genetic polymorphisms and EH risk}

Risk prediction of 14 polymorphisms related to $\mathrm{EH}$ was evaluated under five genetic models of inheritance. Table 3 shows the results obtained from the best genetic model for each SNP. All variants were in Hardy-Weinberg equilibrium (Table 3). However, SLC4A2 rs2303934 was excluded due its low MAF (3.2) (Table 3). The remaining 13 genetic variants were included for further analysis.

Only two showed a statistically significant increase in EH risk $(p<0.05)$, namely, the $A D D 1$ rs4961 variant in the recessive model $(\mathrm{OR}=2.379 ; 95 \% \mathrm{CI}: 1.135-4.985 ; p=0.018)$ and the GNB3 rs5443 variant in the dominant model $(\mathrm{OR}=$ 1.275 ; 95\% CI: 1.042-1.559; $p=0.018$ ) (Table 3). Additionally,

Table 1. Baseline Characteristics of the Population

\begin{tabular}{|c|c|c|c|c|}
\hline Variables & Total $(\mathrm{n}=1641)$ & Hypertensives $(\mathrm{n}=848)$ & Controls $(\mathrm{n}=793)$ & $\mathrm{p}$ Value \\
\hline Age, years & $50.6 \pm 8.1$ & $50.8 \pm 8.1$ & $50.3 \pm 8.2$ & 0.212 \\
\hline Male sex, $n(\%)$ & $819(49.9)$ & $439(51.8)$ & $380(47.9)$ & 0.119 \\
\hline Sedentary life, $n(\%)$ & $887(54.1)$ & $476(56.1)$ & $411(51.8)$ & 0.080 \\
\hline Alcohol, $n(\%)$ & $627(38.2)$ & $350(41.3)$ & 277 (26.6) & 0.008 \\
\hline Smoking, $n(\%)$ & $375(22.9)$ & $164(19.3)$ & $211(26.6)$ & $<0.0001$ \\
\hline $\mathrm{BMI}, \mathrm{kg} / \mathrm{m}^{2}$ & $27.7 \pm 4.9$ & $29.2 \pm 5.2$ & $26.2 \pm 4$ & $<0.0001$ \\
\hline Obesity, $n(\%)$ & $441(26.9)$ & $326(38.4)$ & $115(14.5)$ & $<0.0001$ \\
\hline Diabetes, $n(\%)$ & $169(10.3)$ & $134(15.8)$ & $35(4.4)$ & $<0.0001$ \\
\hline $\mathrm{SBP}, \mathrm{mmHg}$ & $134.4 \pm 20.4$ & $147.2 \pm 18.9$ & $120.7 \pm 10.9$ & $<0.0001$ \\
\hline $\mathrm{DBP}, \mathrm{mmHg}$ & $84.5 \pm 12.1$ & $91.1 \pm 11.7$ & $77.4 \pm 7.7$ & $<0.0001$ \\
\hline Heart rate, bpm & $72.1 \pm 11.8$ & $73.1 \pm 12.2$ & $71 \pm 11.2$ & $<0.0001$ \\
\hline
\end{tabular}

Continuous variables are expressed by mean \pm standard deviation.

Statistically significant for $p<0.05$.

BMI, body mass index; SBP, systolic blood pressure; DBP, diastolic blood pressure; bpm, beats per minute. 
Table 2. Biochemical Characteristics of the Population

\begin{tabular}{lcccc}
\hline Variables & Total $(\mathrm{n}=1641)$ & Hypertensives $(\mathrm{n}=848)$ & Controls $(\mathrm{n}=793)$ & $\mathrm{p}$ Value \\
\hline Hemoglobin, g/dL & $14.3(9.6-18.2)$ & $14.4(9.6-18.2)$ & $14.2(10.1-17.6)$ & $<0.0001$ \\
Platelets, $10^{3} / \mu \mathrm{L}$ & $229(23-664)$ & $233(23-664)$ & $227(65-544)$ & 0.206 \\
Leucocytes, $10^{3} / \mu \mathrm{L}$ & $6.4(2.1-18)$ & $6.6(2.9-18)$ & $6.2(2.1-16.6)$ & $<0.0001$ \\
Fibrinogen, $\mathrm{mg} / \mathrm{dL}$ & $362(179.2-874)$ & $371(179.2-874)$ & $355.3(224-688)$ & $<0.0001$ \\
Glucose, $\mathrm{mg} / \mathrm{dL}$ & $95(66-364)$ & $98(70-360)$ & $93(66-364)$ & $<0.0001$ \\
Lipoprotein $(\mathrm{a}), \mathrm{mg} / \mathrm{dL}$ & $16.3(0.6-236)$ & $16.6(0.6-236)$ & $16.2(0.8-198.2)$ & 0.730 \\
Cholesterol, mg/dL & $207(107-370)$ & $208.5(115-344)$ & $206(107-370)$ & 0.193 \\
HDL, mg/dL & $48(17.2-111.7)$ & $46.9(17.2-103)$ & $49(20.8-111.7)$ & $<0.0001$ \\
LDL, mg/dL & $131(37.7-269)$ & $131(37.7-269)$ & $131(42-260)$ & 0.944 \\
Triglycerides, mg/dL & $110(21-1098)$ & $119(29-1098)$ & $99(21-688)$ & $<0.0001$ \\
Apolipoprotein B, mg/dL & $104(3.9-232)$ & $106.6(5.1-205)$ & $99.7(3.9-232)$ & $<0.0001$ \\
hs-CRP, mg/dL & $0.22(0.01-18.51)$ & $0.25(0.02-13.1)$ & $0.19(0.01-18.51)$ & $<0.0001$ \\
\hline
\end{tabular}

Biochemical variables are presented by median (minimum-maximum)

Statistically significant for $p<0.05$.

HDL, high-density lipoprotein; LDL, low-density lipoprotein; hs-CRP, high-sensitivity C-reactive protein.

the $A C E$ rs4343 was marginally significant being at the threshold of significance $(p=0.053)$ with an OR of $1.148(95 \%$ CI: 0.999-1.321) (Table 3).

\section{Gene-environment interactions and the EH risk}

MDR software was employed to analyze the interaction between the three mentioned variants (ADD1 rs4961, GNB3 rs5443, and $A C E$ rs4343) and five nongenetic risk factors for EH (sedentary lifestyle, obesity, diabetes, smoking, and alcohol habits) (Table 4). The best association model included diabetes, obesity, and ADD1 rs4961 polymorphism, which showed a maximal testing accuracy of 0.638 and a crossvalidation consistency of 10 out of 10 (Table 4). These results indicate that $A D D 1$ rs4961 interacts with diabetes and obesity in a synergistic way with a 3.725-fold greater risk of $\mathrm{EH}$
(Table 4). Consequently this model was chosen as the overall best MDR model, which was significant at a level of 0.0002 , indicating overall a better performance by 2 out of 10,000 permutations and was thus unlikely under the null hypothesis of null association.

To test the validity of the MDR method, we further conducted a logistic regression analysis that combined the two individually significant polymorphisms rs4961 and rs4343, the confounding factors (sedentary lifestyle, smoking, and alcohol habits), and the best interaction MDR model (diabetes, obesity, and $A D D 1 \mathrm{rs} 4961$ ) (Table 5). The results confirmed that the interaction between diabetes, obesity, and $A D D 1$ rs4961 showed a significant association of 10 -fold increased $\mathrm{EH}$ risk (95\% CI: 2.336-43184; $p=0.002$ ) (Table 5). Furthermore, sedentary lifestyle and alcohol presented a risk for EH of 1.229 and 1.390, respectively, with statistical significance $(p<0.05)$.

Table 3. Risk Prediction of Fourteen Polymorphisms Related to Essential Hypertension in Our Population $(N=1641)$, Selected from the Best Genetic Model of Inheritance

\begin{tabular}{|c|c|c|c|c|c|c|c|c|c|}
\hline Gene & $S N P$ & Chr & Position & Cases & Controls & $O R^{\mathrm{a}}(95 \% C I)$ & $\mathrm{p}$ Value & $\begin{array}{c}M A F \\
(\%)\end{array}$ & $\begin{array}{c}\mathrm{p} \text { Value } \\
H W\end{array}$ \\
\hline$A C E \mathrm{I} / \mathrm{D}$ & rs4340 & 17 & $61,565,892$ & 65.7 & 62.8 & $1.137(0.985-1.312)^{\mathrm{b}}$ & $0.079^{\mathrm{b}}$ & 34.3 & 0.110 \\
\hline$A C E 2350 \mathrm{~A} / \mathrm{G}$ & rs4343 & 17 & $63,488,670$ & 58.4 & 55.1 & $1.148(0.999-1.321)^{\mathrm{c}}$ & $0.053^{\mathrm{c}}$ & 41.6 & 0.721 \\
\hline$A G T \mathrm{~T} / \mathrm{M}(\mathrm{T} 174 \mathrm{M})$ & rs4762 & 1 & $230,710,231$ & 30.7 & 29.6 & $1.198(0.941-1.525)^{\mathrm{d}}$ & $0.143^{\mathrm{d}}$ & 30.7 & 0.790 \\
\hline$A G T \mathrm{M} / \mathrm{T}(\mathrm{M} 235 \mathrm{~T})$ & rs699 & 1 & $230,710,048$ & 43.5 & 42.1 & $1.117(0.909-1.373)^{\mathrm{d}}$ & $0.291^{\mathrm{d}}$ & 43.5 & 0.766 \\
\hline$A T 1 R \mathrm{~A} / \mathrm{C}$ & rs5186 & 3 & $148,742,201$ & 24.8 & 24.0 & $1.094(0.900-1.331)^{\mathrm{d}}$ & $0.366^{\mathrm{d}}$ & 24.8 & 0.097 \\
\hline$C Y P 11 B 2 \mathrm{C} / \mathrm{T}$ & rs1799998 & 8 & $142,918,184$ & 55.5 & 55.5 & $0.927(0.724-1.187)^{\mathrm{d}}$ & $0.548^{\mathrm{d}}$ & 44.5 & 0.823 \\
\hline CYP17A1 T/C & rs11191548 & 10 & $103,086,421$ & 10.7 & 10.3 & $1.150(0.473-2.794)^{\mathrm{e}}$ & $0.757^{\mathrm{e}}$ & 10.7 & 0.629 \\
\hline$A D D 1 \mathrm{G} / \mathrm{T}$ & rs4961 & 4 & $2,904,980$ & 16.3 & 14.7 & $2.379(1.135-4.985)^{\mathrm{f}}$ & $0.018^{\mathrm{f}}$ & 16.3 & 0.550 \\
\hline$G N \beta 3 \mathrm{C} / \mathrm{T}$ & rs5443 & 12 & $6,845,711$ & 40.7 & 38.3 & $1.275(1.042-1.559)^{\mathrm{d}}$ & $0.018^{\mathrm{d}}$ & 40.7 & 0.140 \\
\hline$A D R \beta 1 \mathrm{R} / \mathrm{G}(\mathrm{R} 389 \mathrm{G})$ & rs 1801253 & 10 & $114,045,297$ & 30.7 & 29.6 & $1.089(0.897-1.322)^{\mathrm{d}}$ & $0.387^{\mathrm{d}}$ & 30.7 & 0.213 \\
\hline$A D R \beta 2 \mathrm{R} / \mathrm{G}(\mathrm{R} 16 \mathrm{G})$ & rs 1042713 & 5 & $148,826,877$ & 55.8 & 57.8 & $0.925(0.805-1.062)^{\mathrm{b}}$ & $0.268^{\mathrm{b}}$ & 44.2 & 0.716 \\
\hline$S C N N 1 G \mathrm{~A} / \mathrm{G}$ & rs5718 & 16 & $23,182,544$ & 56.8 & 55.7 & $1.170(0.926-1.478)^{\mathrm{d}}$ & $0.187^{\mathrm{d}}$ & 43.2 & 0.014 \\
\hline$S L C 4 A 2 \mathrm{C} / \mathrm{T}$ & rs2303934 & 7 & $150,767,540$ & 3.2 & 2.8 & $1.168(0.769-1.775)^{\mathrm{d}}$ & $0.467^{\mathrm{d}}$ & 3.2 & 0.021 \\
\hline$A T P 2 B 1 \mathrm{~A} / \mathrm{G}$ & rs2681472 & 12 & $89,615,182$ & 15.3 & 15.1 & $0.780(0.433-1.405)^{\mathrm{f}}$ & $0.407^{\mathrm{f}}$ & 15.3 & 0.745 \\
\hline
\end{tabular}

${ }^{\mathrm{a} O R}$ from the best model.

${ }^{\mathrm{b}}$ Multiplicative model.

${ }^{c}$ Additive model.

${ }^{\mathrm{d}}$ Dominant model.

${ }^{\mathrm{e}}$ Codominant model.

${ }_{\mathrm{f}}^{\mathrm{C}}$ Recessive model.

OR, odds ratio; SNP, single nucleotide polymorphism; Chr, chromosome; MAF, minor allele frequency; HW, Hardy-Weinberg using $p<0.004$ after Bonferroni correction; CI, confidence interval. 
Table 4. Best Models to Analyze Gene-Environment Interactions By Multifactorial Dimensionality Reduction

\begin{tabular}{lccccccc}
\hline & $\begin{array}{c}\text { Training } \\
\text { balanced } \\
\text { accuracy }\end{array}$ & $\begin{array}{c}\text { Training } \\
\text { odds ratio }\end{array}$ & $\begin{array}{c}\text { Training } \\
\text { p-value }\end{array}$ & $\begin{array}{c}\text { Testing } \\
\text { balanced } \\
\text { accuracy }\end{array}$ & $\begin{array}{c}\text { Testing } \\
\text { odds ratio }\end{array}$ & $\begin{array}{c}\text { Testing } \\
\text { p-value }\end{array}$ & $\begin{array}{c}\text { CV } \\
\text { consistency }\end{array}$ \\
\hline $\begin{array}{l}\text { Obesity } \\
\text { Diabetes; obesity }\end{array}$ & 0.620 & $3.682(2.855-4.748)$ & $<0.0001$ & 0.620 & $3.682(1.717-7.900)$ & 0.0005 & $10 / 10$ \\
$\begin{array}{c}\text { Diabetes; obesity; } \\
\quad \text { ADDI }\end{array}$ & 0.638 & $3.726(2.936-4.728)$ & $<0.0001$ & 0.635 & $3.726(1.823-7.614)$ & 0.0002 & $10 / 10$ \\
$\begin{array}{c}\text { Diabetes; obesity; } \\
\text { smoking; } \text { ADD1 }\end{array}$ & 0.639 & $3.952(3.1045-4.711)$ & $<0.0001$ & 0.638 & $3.725(1.842-7.535)$ & 0.0002 & $10 / 10$ \\
\hline
\end{tabular}

${ }^{a}$ Overall best combination model with highest training balanced accuracy, highest testing accuracy, and best CV consistency. $\mathrm{CV}$, cross validation.

Smoking appeared with a protective effect for $\mathrm{EH}(\mathrm{OR}=0.615$ 95\% CI 0.485-0.780; $p<0.0001)$.

Because obesity appeared to be an important risk factor for EH development in our MDR analysis, we have further done a crossover analysis (Table 6) and a stratified analysis (Table 7) to investigate whether the obesity or ADD1 rs4961 represented the greatest effect on the EH risk.

Results showed that both obesity and the TT genotype of $A D D 1$ rs4961 greatly increased the susceptibility to hypertension, but obesity seems to have the major effect $(\mathrm{OR}=5.936$ vs. $\mathrm{OR}=2.638)$ (Table 6).

In the stratified analysis, results showed that on obese individuals, the $A D D 1$ rs4961 TT genotype had no significant association with EH $(p=0.546)$. On the other hand, the TT genotype showed a significant increase in EH risk in nonobese individuals $(\mathrm{OR}=2.648,95 \%$ CI: 1.125-6.236; $p=0.021$ ), indicating that the risk for this polymorphism is greater when the person is nonobese (Table 7). The effect of obesity is much stronger than the existence of the ADD1 variant; however, an interaction between the two variables synergistically increases the risk of EH.

\section{Discussion}

It is well established that both genetic and environmental factors contribute to the regulation and maintenance of BP. With the beginning of the human genome project and the International HapMap Project, SNPs have become increasingly prominent in studies of both multifactorial and multigenomic diseases (Kuneš and Zicha, 2009).

In the present work, there were three polymorphic variants, which were significantly associated to EH individually- rs4961, rs5443, and rs4343, this last on the threshold of significance. ADD1 rs4961 was selected in the three-factor model along with obesity and diabetes as the best association model to predict EH. The four-factor model that included obesity, diabetes, $A D D 1$ rs4961, and ACE rs4343 was also selected but had a slightly lower testing balanced accuracy and a weak cross-validation consistency.

The ADD1 G614T polymorphism (rs4961) results in the amino acid substitution of glycine by tryptophan (Gly460Trp), which is reported to be associated with a salt-sensitive form of hypertension. Cusi et al. (1997) found significant linkage of the alpha-adducin locus to EH $(p=0.0003)$ and greater sensitivity to changes in sodium balance among patients with the mutant allele, suggesting that alpha-adducin is associated with a salt-sensitive form of EH (Cusi et al., 1997). However, epidemiological studies have shown that the contribution of $A D D 1$ Gly460Trp mutation to hypertension varies among different ethnic groups. A positive association of this genetic variant with $\mathrm{EH}$ has been confirmed in some studies (Tamaki et al., 1998; Barlassina et al., 2000; Ju et al., 2003; Li et al., 2012), but not in others (Larson et al., 2000; Niu et al., 2010).

As far as we know, this study is the first attempt to establish the interaction between polymorphic variants associated with $\mathrm{EH}$ and several environmental risk factors, in the susceptibility to develop EH, in a cohort of Southern European origin. The interaction between $A D D 1$ polymorphism and nongenetic risk factors, such as obesity and diabetes was identified as the overall optimum synergistic model by using a promising data-mining analytical method, MDR. This method has been successfully applied to detect high-order gene-gene and gene-environment interaction (Guy et al., 2010).

Table 5. Logistic Regression Analysis with Multifactorial Dimensionality Reduction Best Model and Confounding Variables

\begin{tabular}{lcccccc}
\hline Variables & $B$ & $S E$ & Wald & $d f$ & Odds ratio $(95 \%$ CI) & $\mathrm{p}$ Value \\
\hline Smoking & -0.487 & 0.121 & 16.105 & 1 & $0.615(0.485-0.780)$ & $<0.0001$ \\
Sedentary life & 0.206 & 0.101 & 4.190 & 1 & $1.229(1.009-1.497)$ & 0.041 \\
Alcohol & 0.329 & 0.104 & 9.957 & 1 & $1.390(1.133-1.705)$ & 0.002 \\
ADDI (GT + TT) $\times$ Diabetes & 2.307 & 0.744 & 9.611 & 1 & $10.044(2.336-43.184)$ & 0.002 \\
$\quad \begin{array}{l}\text { (Yes) } \times \text { Obesity (Yes) } \\
\text { Constant }\end{array}$ & -0.081 & 0.086 & 0.891 & 1 & 0.922 & 0.345 \\
\hline
\end{tabular}

Forward Wald method (SPSS vs. 19.0) with all variables staying in the model.

Statistically significant for $p<0.05$.

$\mathrm{B}$, beta coefficient; SE, standard error; df, degrees of freedom. 
Table 6. Crossover Analysis Between Obesity AND ADD1 GENOTYPES

\begin{tabular}{lcrrcc}
\hline Obesity & ADD1 & Cases & Controls & OR $(95 \%$ CI $)$ & p Value \\
\hline No & GG & 373 & 492 & & $\overline{-}^{\mathrm{a}}$ \\
& GT & 133 & 178 & $0.986(0.759-1.281)$ & 0.913 \\
& TT & 16 & 8 & $2.638(1.117-6.230)$ & 0.022 \\
Yes & GG & 223 & 78 & $3.771(2.819-5.044)$ & $<0.0001$ \\
& GT & 94 & 35 & $3.543(2.349-5.342)$ & $<0.0001$ \\
& TT & 9 & 2 & $5.936(1.275-27.635)$ & 0.010 \\
\hline
\end{tabular}

Statistically significant for $p<0.05$.

${ }^{\mathrm{a}} \mathrm{OR}$ and $p$-values obtained by the genotype frequency using the $A D D 1$ GG genotype as reference among the nonobese individuals.

Such best interaction cannot be overlooked because in obesity there are a variety of endocrine, genetic, and metabolic mechanisms linked to each other that include insulin resistance, hyperinsulinemia, increased serum aldosterone levels, salt sensitivity, and expanded plasma volume with increased peripheral vascular resistance, which can lead to EH. Since BMI represents the internal metabolic and physiological environment that plays a key role in the development of high BP (Feng et al., 2012), and ADD1 rs4961 is one of the most important targets for salt sensitivity and expanded plasma volume, it is not surprising that their interaction may play an important role in the susceptibility to hypertension. However, the exact pathophysiological mechanisms that contribute to this association require further study.

On the other hand, it is now established that insulin resistance, which predicts type 2 diabetes, also has a role in the development of hypertension (Sowers, 2004; Conen et al., 2007). Indeed, hypertension and diabetes substantially share common pathways, such as obesity, inflammation, oxidative stress, and insulin resistance.

In our case-control study, diabetes, obesity, alcohol consumption, and $A D D 1$ TT genotype were confirmed as independent risk factors for $\mathrm{EH}$ by multivariate logistic regression. Curiously, smoking habits appear to be a protector factor. A likely explanation of these apparent protective effects of smoking on hypertension might be related to the fact that physicians had likely advised their hypertensive patients to quit smoking, so that the proportion of smoking hypertensives is lower than the proportion of nonsmoking ones.

Further research on gene-gene and gene-environment interaction with well-designed studies containing large sample size and multiple genetic polymorphisms and environmental risk factors are needed to provide more precise evidence for the issue.

Table 7. Stratified Analysis with the Interaction Between Obesity and ADD1 Genotypes

\begin{tabular}{lcrrcr}
\hline Obesity & ADD1 & Cases & Controls & OR $(95 \%$ CI $)$ & $\mathrm{p}$ Value \\
\hline No & GG & 373 & 492 & & \\
& GT & 133 & 178 & $2.648(1.125-6.236)$ & 0.021 \\
& TT & 16 & 8 & & \\
Yes & GG & 223 & 78 & & \\
& GT & 94 & 35 & $1.604(0.341-7.536)$ & 0.546 \\
& TT & 9 & 2 & & \\
\hline
\end{tabular}

OR and $p$-values obtained by the recessive model (TT vs. GG $+\mathrm{GT}$ ). Statistically significant for $p<0.05$.

\section{Strength and limitations}

One limitation of the study was that only 13 polymorphisms of relevant genes for EH were analyzed, and it is of added interest to explore other candidate genes within different metabolic axes and other emerged from GWAS with yet unknown pathophysiological mechanisms.

Other limitation to the approach is that MDR method does not have a way to adjust for covariate effects, such as age, gender, alcohol, and smoking status, an often necessary step to obtain an unconfounded SNP interactions outcome. For this reason, we used a second logistic regression with the MDR best model adjusted for confounded variables and so we were able to confirm the independence of this interaction.

A strength of our study was the specificity of our population, which is supposed to be a genetically homogeneous Southern European Caucasian population (Brehm et al., 2003; Gonçalves et al., 2005). The use of genetically isolated populations has been particularly valuable for mapping rare recessive disorders, or more complex disorders due to a relatively uniform genetic background of the populations (Kristiansson et al., 2008). Some culturally and genetically isolated populations have a more similar way of living, eating habits, and natural environment that reduces environmental variation (Jorde and Wooding, 2004).

\section{Conclusion}

According to our findings, the evolution to EH may actually be affected by the interaction of $A D D 1$ rs4961, obesity, and type 2 diabetes in our population. In patients carrying this polymorphism, involved in salt handling, it will be of utmost importance the modification of lifestyle.

\section{Acknowledgment}

This study was supported by the European Regional Development Fund's Operational Program for the Enhancement of Economic Potential and Territorial Cohesion for the Autonomous Region of Madeira (INTERVIR+).

The authors are very grateful to Elsa Sousa who performed the phone calls to the subjects and all the administrative procedures.

\section{Author Disclosure Statement}

No competing financial interests exist.

\section{References}

Altshuler D, Daly MJ, Lander ES (2008) Genetic mapping in human disease. Science 322:881-888.

Barlassina C, Norton GR, Samani NJ, et al. (2000) Alphaadducin polymorphism in hypertensives of South African ancestry. Am J Hypertens 13:719-723.

Brehm A, Pereira L, Kivisild T, et al. (2003) Mitochondrial portraits of the Madeira and Açores archipelagos witness different genetic pools of its settlers. Hum Genet 114:77-86.

Carretero OA, Oparil S (2000) Essential hypertension. Part I: definition and etiology. Circulation 101:329-335.

Conen D, Ridker PM, Mora S, et al. (2007) Blood pressure and risk of developing type 2 diabetes mellitus: the Women's Health Study. Eur Heart J 28:2937-2943. 
Cowley AW, Jr. (2006) The genetic dissection of essential hypertension. Nat Rev Genet 7:829-840.

Cusi D, Barlassina C, Azzani T, et al. (1997) Polymorphisms of alpha-adducin and salt sensitivity in patients with essential hypertension. Lancet 349:1353-1357.

Ehret GB, Morrison AC, O'Connor AA, et al. (2008) Replication of the Wellcome Trust genome-wide association study of essential hypertension: the Family Blood Pressure Program. Eur J Hum Genet 16:1507-1511.

Ehret GB, Munroe PB, Rice KM, et al. (2011) Genetic variants in novel pathways influence blood pressure and cardiovascular disease risk. Nature 478:103-109.

European Society of Hypertension-European Society of Cardiology Guidelines Committee (2003) 2003 European society of Hypertension-European society of cardiology guidelines for the management of arterial hypertension. J Hypertens 21:1011-1053.

Expert Committee on the Diagnosis and Classification of Diabetes Mellitus (2003) Report of the Expert Committee on the diagnosis and classification of diabetes mellitus. Diabetes Care 26 Suppl 1:S5-S20.

Feng RN, Zhao C, Wang C, et al. (2012) BMI is strongly associated with hypertension, and waist circumference is strongly associated with type 2 diabetes and dyslipidemia, in northern Chinese adults. J Epidemiol 22:317-323.

Gonçalves R, Freitas A, Branco M, et al. (2005) Y-chromosome lineages from Portugal, Madeira and Açores record elements of Sephardim and Berber Ancestry. Ann Human Genet 69:443-454.

Guy J, Andrew AS, Andrews P, et al. (2010) A simple and computationally efficient sampling approach to covariate adjustment for multifactor dimensionality reduction analysis of epistasis. Hum Hered 70:219-225.

Ji L, Cai X, Zhang L, et al. (2013) Association between polymorphisms in the renin-angiotensin-aldosterone system genes and essential hypertension in the Han Chinese population. PLoS One 8:e72701.

Ji W, Foo JN, O'Roak BJ, et al. (2008) Rare independent mutations in renal salt handling genes contribute to blood pressure variation. Nat Genet 40:592-599.

Jorde L, Wooding SP (2004) Genetic variation, classification and race. Nat Genet 36 Suppl 11:528-533.

Joseph PG, Pare G, Anand SS (2013) Exploring gene-environment relationships in cardiovascular disease. Can J Cardiol 29:37-45.

Ju Z, Zhang H, Sun K, et al. (2003) Alpha-adducin gene polymorphism is associated with essential hypertension in Chinese: a case-control and family-based study. J Hypertens 21:1861-1868.

Kristiansson K, Naukkarinen J, Peltonen L (2008) Isolated populations and complex disease gene identification. Genome Biol 9:109.

Kuneš J, Zicha J (2009) The interaction of genetic and environmental factors in the etiology of hypertension. Physiol Res 58 Suppl 2:S33-S41.

Larson N, Hutchinson R, Boerwinkle E (2000) Lack of association of 3 functional gene variants with hypertension in African Americans. Hypertension 35:1297-1300.

Levy D, Ehret GB, Rice K, et al. (2009) Genome-wide association study of blood pressure and hypertension. Nat Genet 41:677-687.
Levy D, Larson MG, Benjamin EJ, et al. (2007) Framingham Heart Study $100 \mathrm{~K}$ project: genome-wide associations for blood pressure and arterial stiffness. BMC Med Genet 8 Suppl 1:S3.

Lifton RP, Gharavi AG, Geller DS (2001) Molecular mechanisms of human hypertension. Cell 104:545-556.

Li K, Liang Y, Sun Y, et al. (2012) The relationship between polymorphisms at 17 gene sites and hypertension among the aboriginal Tibetan people. Biomed Environ Sci 25: 526-532.

Mein CA, Caulfield MJ, Dobson RJ, et al. (2004) Genetics of essential hypertension. Hum Mol Genet 13 Spec No 1:R169R175.

National Institutes of Health, National Heart, Lung, and Blood Institute North American Association for the Study of Obesity (2000) The practical guide: identification, evaluation, and treatment of overweight and obesity in adults. NHLBI Obesity Education Initiative, NIH Publication Number: 00-4084.

Niu WQ, Zhang Y, Ji KD, et al. (2010) Lack of association between alpha-adducin G460W polymorphism and hypertension: evidence from a case-control study and a metaanalysis. J Hum Hypertens 24:467-474.

Okamoto K, Aoki K (1963) Development of a strain of spontaneously hypertensive rats. Jpn Circ J 27:282-293.

Oparil S, Zaman A, Calhoun DA (2003) Pathogenesis of hypertension. Ann Intern Med 139:761-776.

Sowers JR (2004) Insulin resistance and hypertension. Am J Physiol Heart Circ Physiol 286:H1597-H1602.

Tamaki S, Iwai N, Tsujita Y, et al. (1998) Polymorphism of alpha-adducin in Japanese patients with essential hypertension. Hypertens Res 21:29-32.

The Department of Health, Government of Australia (2014) Physical Activity and Sedentary Behavior Guidelines. Available at www.health.gov.au/internet/main/publishing.nsf/content/healthpubhlth-strateg-phys-act-guidelines (accessed August 24, 2017).

Wellcome Trust Case Control Consortium (2007) Genomewide association study of 14,000 cases of seven common diseases and 3,000 shared controls. Nature 447:661-678.

Yang CH, Lin YD, Yang CS, et al. (2015) An efficiency analysis of high-order combinations of gene-gene interactions using multifactor-dimensionality reduction. BMC Genomics 16:489.

Zuoguang W, Xiaoyun P, Yongxiang W, et al. (2013) Neglect of several important indexes during the study of human arterial hypertension. J Clin Hypertens 15:11.

Address correspondence to: Ana Célia Sousa, MD Unidade de Investigação Hospital Doutor Nélio Mendonça Avenida Luís de Camões, no. 57 Funchal, Madeira 9004-514 Portugal E-mail: anacelia.bett@gmail.com 